\begin{tabular}{l|l} 
Revista Educación \\
ISSN: 0379-7082 \\
ISSN: 2215-2644 \\
revedu@gmail.com \\
Universidad de Costa Rica \\
Costa Rica
\end{tabular}

\title{
La herencia cultural didáctica: Un estudio relativo al concepto de área en la formación docente
}

Morales Paredes, Hernán; González Méndez, Ricardo

La herencia cultural didáctica: Un estudio relativo al concepto de área en la formación docente

Revista Educación, vol. 43, núm. 2, 2019

Universidad de Costa Rica, Costa Rica

Disponible en: http://www.redalyc.org/articulo.oa?id=44058158021

DOI: https://doi.org/10.15517/revedu.v43i2.31883

Esta obra está bajo una Licencia Creative Commons Atribución-NoComercial-SinDerivar 3.0 Internacional. 


\title{
La herencia cultural didáctica: Un estudio relativo al concepto de área en la formación docente
}

\author{
A Didactic Cultural Heritage: A Study on Mathematical Teacher Education and Teaching the Concept of Area
}

Hernán Morales Paredes

Facultad de Educación, Universidad Católica de la

Santísima Concepción, Chile

hmorales@ucsc.cl

iD http://orcid.org//0000-0002-9683-0927

Ricardo González Méndez

Facultad de Educación, Universidad Católica de la

Santísima Concepción, Chile

rgonzalez@ucsc.cl

(iD http://orcid.org/0000-0002-7319-5561

\author{
DOI: https://doi.org/10.15517/revedu.v43i2.31883
http://www.redalyc.org/articulo.oa?id=44058158021 \\ Redalyc: http://www.redalyc.org/articulo.oa?id=44058158021
}

(D)t//orcid.org/000-0002-7319-5561

Recepción: 28 Diciembre 2017

Aprobación: 21 Mayo 2019

\section{RESUMEN:}

En este artículo se presenta un estudio en el cual interesa observar la herencia cultural didáctica respecto del concepto área de un cuadrilátero que posee el estudiantado que ingresa a estudiar la carrera de Pedagogía Media en Matemática en la Universidad Católica de la Santísima Concepción (UCSC). Se desea dar cuenta de lo que el alumnado declara cuando se le solicita planificar una unidad didáctica para enseñar el concepto de área de un cuadrilátero. Con esta información se observa la herencia cultural didáctica otorgada por el proceso de enseñanza en el sistema educativo escolar chileno y propone un punto referencial para monitorear y comparar las modificaciones que se producen al efectuar el proceso de formación en la UCSC. Para desarrollar la investigación se contrastaron las propuestas del estudiantado, con elementos fundamentados en la Teoría de las Situaciones Didácticas (TSD) planteada por Guy Brousseau (1986). La forma de enseñanza más presente implica la presentación de las estructuras axiomáticas para luego resolver problemas de tipo reproductivo y piensan la enseñanza como una forma tradicional de organización. Sin embargo, hay estudiantes que presentan una forma de enseñanza más cercana a aplicaciones del mundo real, conlleva procesos de más diálogo entre docentes y alumnado, formas de representación más diversas de objetos geométricos, ejercicios de tipo reflexivo y presencia de devolución. Además, este alumnado muestra mayor dominio conceptual del concepto de área. Los resultados denotan que repite sus formas de enseñanza de acuerdo con cómo se les enseñó en el aula escolar.

Palabras clave: Formación de Docentes de Matemática, Didáctica de la Matemática, Teoría de las Situaciones Didácticas, Herencia Cultural Didáctica.

\section{Abstract:}

This article addresses how the concept of finding the area of a quadrilateral has been traditionally taught to student teachers majoring in Middle School Mathematics Education at the Catholic University of Santísima Concepción (UCSC) in Chile. It is important to note that Education Majors already possess a notion as to how to teach the concept of finding the area of a quadrilateral which we refer to here as their didactic cultural heritage. This study observes didactic cultural heritage as a product of the Chilean Educational School System and proposes a baseline to monitor and compare results among these students at UCSC and compare student views with fundamental elements of Didactic Theoretical Situations (DTS) according to Guy Brousseau (1986). Current teaching strategies are based on axiomatic structures and reproductive problem resolution and consider teaching as based on a traditional organizational method. However, students have also proposed more practical teaching methods applicable to real-life scenarios which involve increased teacher-student interaction and additional ways of representing diverse geometric objects, reflexive type exercises and active "devolution" alluding to a more conceptual mastery of area. The results conclude that teaching methods tend to be repeated and Education Majors, in this case, focusing on Middle School Math, will ultimately teach a concept according to how they were taught that same concept in the classroom when they were students in Middle School.

KEYWORDS: Mathematics Teacher Education, Mathematics Teaching Methodology, Theory of Didactical Situations, Didactic Cultural Heritage. 


\section{INTRODUCCIÓN}

En Chile, son las universidades las encargadas de formar un bueno y exitoso personal docente (Ávalos, 2004). Sin embargo, en este proceso de formación hay una variable relevante, esta consiste en que el estudiantado cuando llega a la universidad ya trae una concepción sobre cómo enseñar algún tipo de contenido matemático, y probablemente esa concepción es producto de su experiencia personal como estudiante de una escuela (Winsløw, 2013); a esta concepción se le llama herencia cultural didáctica. La universidad a través del proceso de formación interviene sobre la concepción de enseñanza del alumnado, y esa intervención debería provocar algún tipo de influencia o modificación de su herencia cultural didáctica. Para dar cuenta de esa modificación se ha propuesto averiguar ¿cuáles son las concepciones de enseñanza que trae previamente el estudiantado?; ¿cuál es el referente epistemológico que posee cuando ingresa a la universidad respecto del concepto área de un cuadrilátero?; ¿qué tipo de relaciones declara este estudiantado para el logro de aprendizajes entre el profesorado y el alumnado?

Desde el año 2012 se ha dictado la asignatura de Didáctica de la Geometría en el Programa de Formación de Docentes de Pedagogía en Educación Media en Matemática (Facultad de Educación, 2011), en la Universidad Católica de la Santísima Concepción (UCSC), Chile, en el cual se forman docentes especialistas en matemáticas para enseñar a escolares desde los 12 a los 17 años de edad de las escuelas chilenas. Ahí se ha podido observar cómo transcurre la modificación del sistema de prácticas del estudiantado. Entre los referentes concretos de la existencia de la herencia cultural didáctica, está lo que se observó en una entrevista efectuada a 12 docentes del sistema educacional chileno que se encontraban trabajando en la enseñanza de la geometría en sus escuelas. Cuando al grupo docente se le pidió proponer actividades de enseñanza, y observar la propuesta del concepto de área de un cuadrilátero que ellos declaraban, se observó que se enfocaban en la fórmula de cálculo del área en la cual la forma recurrente en que se determina el concepto de área es bajo la condición del cálculo lado a por lado $b, a \times b$. En las relaciones entre docentes y alumnado, las actividades de enseñanza declaradas les solicitan que memoricen las fórmulas de área y luego las apliquen.

Considerando esos elementos iniciales, este artículo tiene como objetivo dar cuenta de la herencia cultural didáctica y del sistema de prácticas que poseen el estudiantado al ingresar a un proceso de formación de docentes, que es parte de la investigación desarrollada. Para eso se propuso una contrastación entre las respuestas del alumnado y la Teoría de las Situaciones Didácticas de Brousseau (Brousseau, 1986, 2007, 2013), que fundamenta el proceso de formación de docentes en la asignatura de Didáctica de la Geometría (Facultad de Educación, 2012). Los elementos presentes y ausentes en esta contrastación entre lo declarado por el estudiantado y elementos de la TSD fueron observados de acuerdo con el contenido de referencia de área de un cuadrilátero, señalado en el programa de estudio propuesto por el Ministerio de Educación (MINEDUC, 2012).

Los elementos contextuales que se han indicado, justificaron llevar adelante esta investigación, pues puede entregar orientaciones para obtener información relevante respecto de la influencia de la formación inicial docente sobre la herencia cultural didáctica de estudiantes de pedagogía en matemática; observar si existe coherencia con el proceso de formación vivido en la universidad, establecer su impacto o debilidades considerando lo determinado por Winsløw (2009), y que el proceso de formación se ve frenado o influenciado por las experiencias previas que el estudiantado ha tenido como alumno o alumna en la escuela. Igualmente, junto a la necesidad de retroalimentar el proceso se encuentra la variable de acreditación de carreras de formación de docentes, que es obligatoria en Chile, de tal modo que el estudiantado que se forme en instituciones no acreditadas no podría desempeñarse en el sistema público de educación (CNAChile, 2018). En cierta manera, se obliga a las instituciones universitarias a formar profesionales, que les aseguren a ellos mismos estándares de desempeño adecuados a los requeridos por el sistema, que puede tener efectos sobre sus remuneraciones (MINEDUC, 2004, 2018) y mejores posibilidades contractuales, y a su 
vez, un profesor con mal desempeño puede ser desvinculado de la institución en la que trabaja. Dada estas variables profesionales, es relevante que las universidades comprometan ciertas características en su proceso de formación, asociadas a una buena calidad de la enseñanza, y en este sentido en esta investigación interesa visualizar el impacto de un proceso de formación de docentes en el futuro desempeño profesional hacia un estudiantado que se ha formado en la universidad.

\section{REFERENTE TEÓRICO}

El proceso de formación mencionado es observado a través del modelo de la Teoría Antropológica de lo Didáctico (TAD), en su versión referida a la relación institución ( $\mathrm{RI}$ ), individuo (p) y objeto de conocimiento (o); $R I(p, o)$ (Chevallard, 1999; Winsløw, 2013), que se presenta a través del modelo $R E\left(\mathrm{a}, \mathrm{o}_{1}\right) \rightarrow R U(\mathrm{e}, \ddot{\mathrm{o}})$ $\rightarrow R E\left(\mathrm{ep}, \mathrm{o}_{2}\right)$, (Winsløw, 2009); como el paso de un/a estudiante que inicia su proceso de formación en la universidad $R E\left(\mathrm{a}, \mathrm{o}_{1}\right)$, el cual luego finaliza el curso de Didáctica de la Geometría RU(e,ö), y que posteriormente le permite actuar como profesor/a (sin serlo) en la asignatura de Práctica Pedagógica Profesional $R E\left(\mathrm{ep}, \mathrm{o}_{2}\right)$. En este escrito, $\mathrm{o}_{1}, \mathrm{o}_{\mathrm{y}} \mathrm{o}_{2}$ son objetos de conocimiento matemático que corresponde al concepto de área de un cuadrilátero que el estudiantado posee en distintos momentos. La siguiente Tabla 1 representa los tres momentos que son observados y son parte del proceso de formación en la universidad:

TABLA 1

\section{Representación de la herencia cultural didáctica del estudiantado en distintos momentos}

\begin{tabular}{lll}
\hline RE(a,o1) $\rightarrow$ & $\mathrm{RU}(e, 0) \rightarrow$ & $\mathrm{RE}(e \mathrm{e}, 02)$ \\
\hline $\begin{array}{l}\text { Al inicio del curso de Didáctica de la } \\
\text { Geometría }\end{array}$ & $\begin{array}{l}\text { Al finalizar el curso de Didáctica de la } \\
\text { Geometría }\end{array}$ & Al inicio del curso de Práctica Profesional. \\
\hline
\end{tabular}

Nota: Elaboración propia

En el contexto de este artículo, solo se observa el estado del sistema de prácticas para enseñar el concepto de área de un cuadrilátero inicial $\operatorname{RE}\left(a, o_{1}\right)$.

En el proceso de formación de docentes previsto en la universidad, se estableció, como lo propone (Winsløw, 2009), que el sistema de prácticas asociado a la herencia cultural didáctica traída por el estudiantado que ingresa, necesariamente debía ser modificado, pues el referente experiencial es que el estudiantado utiliza un modelo tradicional que se caracteriza por la fuerte presencia de un método idealista platónico, el cual considera que el alumnado "debe adquirir primero las estructuras fundamentales de las matemáticas de forma axiomática. Se supone que una vez adquirida esta base, será fácil que el alumnado por sí solo pueda resolver las aplicaciones y problemas que se le presenten” (Godino, Batanero y Font, 2003, p. 20). Para la modificación se propuso una estructura que implicaba establecer un modelo de planificación de la enseñanza, y que se fundamentó en el señalado por el Ministerio de Educación de Chile; inicio, desarrollo y cierre (MINEDUC, 2012). Sin embargo, resultaba insuficiente por lo global y general, y porque no logra explicitar elementos de la Didáctica de la Geometría que fundamentaban el programa de formación. Estos elementos eran la Transposición Didáctica (Chevallard, 1999) y la Relación <Sujeto-Medio $>$ (Brousseau, 1986).

En Chile existe una investigación respecto de concepciones alternativas de área y perímetro, desarrollada por Reyes, Dissett y Gormaz (2013) quienes muestran ciertos obstáculos y errores. Muestran que es muy común en el alumnado confundir fórmulas de perímetro y área. Por ejemplo, "un alumno sistemáticamente al calcular el área de rectángulos entrega como resultado el doble de la medida correcta” (p. 165), pues el alumnado al calcular el perímetro de un rectángulo suma la medida de los lados y el resultado lo multiplica por dos, entonces en el cálculo del área ocurre lo mismo; multiplica la medida de los lados y ese resultado lo multiplica erróneamente por dos. Otra concepción alternativa es suponer que si dos rectángulos diferentes 
tienen el mismo perímetro, entonces tiene la misma área o rectángulos que tienen la misma área, pero diferente perímetro.

Otra investigación muestra una intervención en el proceso de formación de docentes para obtener una suficiente y exitosa influencia en los aprendizajes del alumnado en las escuelas. Específicamente durante el año 2014 se desarrolló una con fuertes vínculos hacia la enseñanza de la matemática, titulada Recursos para la formación inicial de docentes (Martínez, 2014), que elaboró una colección de textos para "mejorar la preparación de los futuros docentes para enseñar matemáticas, y para favorecer la implementación de estándares mínimos de formación" (Martínez, 2014, p. 12). Los textos propuestos están diseñados para la formación de un profesor que se prepara para enseñar el currículum escolar chileno de matemáticas, es decir, para enseñar lo que el alumnado debe aprender, tal como está definido en el currículum (Martínez, 2014). El fundamento teórico se centra en el MKT (Mathematical Knowledge for Teaching) que incluye conocimientos disciplinares y conocimientos pedagógicos del contenido (Ball, Hill y Bass, 2005; Ball, Thames y Phelps, 2008).

Entre los resultados de las aplicaciones de los textos, Martínez (2014) explica que se observó una fuerte presencia del conocimiento matemático común en desmedro del conocimiento especializado y del conocimiento pedagógico del contenido. Esta observación se ha repetido en el escenario internacional. Bacon (2009), señala que hay una ausencia de conocimientos didácticos en los procesos prácticos. Del mismo modo, Perrenoud (2000) presentaba un análisis más descriptivo respecto de esta misma ausencia, al establecer que es importante organizar un debate respecto de qué hacer en formación inicial para ser coherente con lo solicitado por la sociedad. En este sentido, en la práctica profesional debiera considerarse el proyecto personal del estudiantado que se forma, compatible con el diseño y los objetivos generales de la formación inicial. De lo anterior, se desprende que los problemas y escenarios de formación de docentes en un contexto internacional son similares a los chilenos y a lo que ocurre en particular en la UCSC en la formación de docentes de Pedagogía en Educación Media en Matemática.

\section{Elementos de la Teoría de las Situaciones Didácticas (TSD)}

La TSD ha sido considerada como relevante en el proceso de formación, pues es un referente que proporciona conceptos y estrategias para comprender y regular la enseñanza de las matemáticas (Morales, 2017). Esta teoría comporta elementos que son claves en el proceso de formación de docentes al cual se adscribe esta investigación. Uno de los elementos es el sistema < sujeto - medio> (Brousseau, 1986, 2013); paradigma <s$\mathrm{m}>$, y que propone que el aprendizaje se produce por adaptación, es decir un individuo aprende al enfrentar a su medio, todo lo que él conoce, y desenvolverse en él, lo cual implica que el aprendizaje se produce a partir de la interacción entre un sujeto (el alumnado) y un medio (Acosta, Monroy, y Rueda, 2010; Brousseau, 1986, 2013; Chavarría, 2006; D'Amore, 2011; D'Amore y Fandiño, 2002; Johsua y Dupin, 2005).

En un sistema educativo formal, el objetivo de aprendizaje que el alumnado debe lograr lo proporciona el profesorado, entonces este modifica el medio creando una situación o tarea y luego el alumnado resolverá esa tarea, adaptándose al medio. Brousseau (1986), apunta que el alumnado aprende adaptándose a un medio que es factor de contradicciones, dificultades, desequilibrios. Ese saber fruto de la adaptación del alumnado, se manifiesta por las respuestas nuevas que son la prueba del aprendizaje, así este aprendizaje se logra cuando en el alumnado aparecen evidencias de adaptaciones deseables (Brousseau, 1986; Chavarría, 2006; D'Amore, 2011; Johsua y Dupin, 2005). La modificación del medio es una acción que ejecuta el profesorado, y esa acción se denomina situación. 


\section{Situación (matemática)}

El aprendizaje de las matemáticas se considera vinculado a una actividad intelectual que consiste en proponer y resolver una situación o ejercicios, tareas o problemas. El profesorado propone algunas tareas o problemas matemáticos y el alumnado los resuelve; al resolverlos construye el conocimiento matemático (Acosta et ál., 2010; Brousseau, 1986, Chavarría, 2006; D'Amore, 2005, 2011; D'Amore y Fandiño, 2002).

Brousseau (2013), indica que una situación forma un sistema que considera las condiciones de uso particular de los conocimientos. Agrega que "una situación se caracteriza en una institución por un conjunto de relaciones y roles recíprocos de uno o más sujetos (estudiantes, docentes, etc.) con un medio, considerando la transformación de este medio según un proyecto" (p. 3). Entonces, la situación comprende los problemas matemáticos que se plantean, las preguntas que efectúa el profesorado, los métodos y técnicas de resolución, la información que se presente con la intención de enseñar; propondrá un problema para que el alumnado se apropie de los conocimientos matemáticos específicos, a través de lo que Brousseau (1986) llama situación adidáctica.

Para los efectos de clasificación del tipo de situaciones, en esta investigación se ha utilizado el marco PISA (OCDE, 2006), el cual establece tres niveles de competencia asociados al tipo de situación. Estos niveles son: reproducción, situaciones que implican representaciones y definiciones estándar; conexión, situaciones que implican construcción de modelos e interpretación de problemas; y reflexión, situaciones con un enfoque matemático original. El mismo marco PISA plantea el ciclo de la matematización en que una situación se inicia con un problema situado en la realidad.

\section{Situación didáctica, situación adidáctica, institucionalización, devolución}

En la TSD de Brousseau (1986), el profesorado es el encargado de crear la intención en el alumnado de desarrollar conceptos y procedimientos matemáticos y además de preparar correctamente el medio. Para cumplir ese rol debe existir la intención de enseñar un conocimiento matemático determinado a un alumno. A esta acción que ocurre en un aula escolar se le denomina situación didáctica. El profesorado, que desea enseñar algún contenido matemático al alumno, no le plantea este contenido comunicándoselo directamente, sino planteándole un problema relacionado al conocimiento matemático y que el alumnado debe resolver por sí solo, con su medio, ya que el aprendizaje de un concepto matemático se produce bajo el paradigma $<s-\mathrm{m}>$. Como se puede percibir, el vínculo entre el alumnado y un medio no tiene ninguna intención didáctica, el alumnado está solo frente al medio, sin el profesorado, y el medio no le enseña al alumno. Este tipo de situación, recibe el nombre de situación adidáctica. En el proceso de crear la intención de que el alumnado acepte la responsabilidad de resolver un problema, lo que hace el profesorado es preparar cuidadosamente un medio con el cual el alumnado pueda interactuar, y un problema que desencadene acciones sobre el medio. Luego de finalizada la situación adidáctica el profesorado debe explicitar las relaciones entre el conocimiento construido y el conocimiento matemático presentado, reconociendo la validez y la utilidad de este nuevo conocimiento, que es una modificación del anterior. A esta etapa Brousseau (2013) la llama institucionalización de un conocimiento. Él la define como "una situación que se presenta por el paso de un conocimiento de una situación de acción, de formulación o de prueba, a un nuevo rol, el de referencia para las utilizaciones futuras" (p. 4). La institucionalización ocurre cuando el profesorado en el aula, frente al curso, ya en una situación didáctica, corrige y valida la o las respuestas presentadas, y comunica el nuevo conocimiento matemático (Johsua y Dupin, 2005).

Cuando se produce la situación adidáctica, podría ocurrir que el alumnado no pueda resolver el problema y no se produzca la validación. Si esto ocurre, el profesorado debe animar y guiar al estudiante para que continúe en el proceso de resolverlo. A esta acción del profesorado se le llama devolución, acción en que "el profesorado 
gestiona a través de una situación didáctica posicionar al alumno como único protagonista en una situación adidáctica" (Brousseau, 2013, p. 5). El profesorado no intercede en la resolución de la situación por parte del alumnado; si lo hiciese, impediría que se produzca un aprendizaje por adaptación. Sin embargo, lo anterior no significa que el profesorado no deba intervenir, sino que su vínculo con el alumnado debe limitarse a animar al alumno a resolver el problema planteado, evitar que renuncie a resolverlo, asegurándose de que el alumnado entienda o comprenda lo que se espera que logre. Aun así, si el alumnado no puede resolver el problema pudiese ocurrir que se presente el efecto Topaze, es decir el profesorado asume la resolución del problema (Brousseau, 2013; Johsua y Dupin, 2005).

\section{El contrato didáctico}

El contrato didáctico sostiene la relación entre el profesorado, el y el conocimiento matemático, y se hace presente a través de las situaciones. Brousseau, (2013) define el contrato didáctico como el "conjunto de obligaciones recíprocas y de sanciones que cada participante de la situación didáctica impone o cree imponer, explícita o implícitamente a los otros y las que le imponen o que él cree que le imponen" (p. 6). D'Amore (2011) plantea que el contrato didáctico se considera en muchas oportunidades como un acto simbólico inicial por medio del cual el alumnado se convierte en sujeto didáctico al interior de una institución escolar. El Contrato Didáctico no se debe confundir con el Contrato Pedagógico; este último hace referencia a reglas de comportamiento conductual en el aula escolar.

\section{Tipos de situaciones: acción, formulación y validación}

La situación de acción es coherente con la situación adidáctica. Se prepara de manera individual, el alumnado está solo con su medio. Esto lo obliga a actuar sobre el medio, creando o planteando un modelo para solucionar el problema, y mejorarlo en la medida que ocurra la retroacción. La situación de acción es "una situación donde el conocimiento del tema se manifiesta por las decisiones, acciones regulares y eficaces sobre el medio" (Brousseau, 2013, p. 3).

La situación de acción exige que el alumnado plantee respuestas las cuales aparezcan como la mejor posibilidad de solución al problema que ha propuesto el profesorado. Considerando que el medio reacciona sobre el alumnado, a través de la retroacción, le permitirán juzgar el resultado de su acción, y de ajustarla sin la intervención del profesorado (Acosta et ál., 2010; Brousseau, 1986; Chavarría, 2006; D'Amore, 2011; Johsua y Dupin, 2005).

Considerando que el contexto en que se plantea el problema ocurre en la situación didáctica, el alumnado no estará solo, sino que también estarán sus compañeros con quienes podrá comentar y comparar la propuesta de solución al problema. Así, el momento que ocurre cuando el alumnado trabaja en grupo con alguno (o algunos) de sus compañeros, se denomina situación de formulación (Johsua y Dupin, 2005), situación que le permite al alumno comparar su respuesta, validarla al interior del grupo y construir una nueva respuesta la cual es la consensuada del grupo. Se requiere de un grupo de estudiantes que interactúen, pues alguno de ellos debe plantear el conocimiento relacionado para que los otros lo conviertan en una decisión válida sobre el medio.

Una tercera situación llamada situación de validación, ocurre cuando cada grupo que se ha formado en el aula presenta la respuesta al problema frente al resto de compañeros que han trabajado también en grupos. Esto permite una comparación entre todos los y las estudiantes del curso, similar a lo que ocurre en una pequeña comunidad científica que contrasta las propuestas o soluciones de un problema (Johsua y Dupin, 2005). 
Estos tres tipos de situaciones fundamentan lo que se denomina Protocolo de Gestión de Aula (Morales, 2017). Se propone que al ejecutar un proceso de enseñanza, el profesorado organice y gestione siguiendo este Protocolo, desarrollando un procedimiento que considera tres etapas secuenciales. La primera etapa es que el profesorado solicita a cada alumno que individualmente resuelva el problema; así el alumnado está solo frente al problema, con sus propios conocimientos, por lo que se produce la acción y retroacción con el medio. Esta etapa se asimila a la situación de acción planteada por (Brousseau, 2013; Johsua y Dupin, 2005). La segunda etapa, el profesorado organiza al curso para que se produzca un trabajo en grupo y ocurra la situación de formulación. Cada grupo habrá elegido su mejor respuesta al problema en un proceso de diálogo y validación grupal. En la tercera etapa, el profesorado elige a un integrante de cada grupo para que exponga la respuesta al problema y así se produce la pequeña comunidad científica que corresponde a la situación de validación. La organización será dirigida por el profesorado, y luego de finalizada la situación de validación, el profesorado efectuará la institucionalización.

En el proceso de formación de docentes, el Protocolo de Gestión de Aula (Morales, 2017) se presenta de manera transversal como un deber ser del profesorado. Eso implica que la clase regida bajo el Protocolo se inicia con una situación didáctica, y que el proceso de resolución de la situación, tarea, ejercicio o problemas, sigue el proceso adidáctico y el profesorado ejecuta la devolución hasta llegar a la institucionalización.

\section{Base epistemológica del concepto de área}

En este trabajo se muestra una conceptualización que se llama tradicional y está asociada a la comprensión del concepto de área como un cálculo algorítmico de la multiplicación de la medida de sus lados a $\mathrm{x}$ b. Sin embargo, en coherencia, en el proceso de formación de docentes en esta investigación, se utiliza el concepto de área como medida de la superficie (Clemens, O'daffer, y Cooney, 1998) entonces es necesario expresarla con su respectiva unidad de medida, que conlleva una información cuantitativa, este concepto se observa en la siguiente Figura 1. La medida implica la asignación de un número a una propiedad física, como resultado de una comparación de dicha propiedad con otra similar tomada como patrón, el cual es adoptado como unidad (Clemens et ál., 1998; MINEDUC, 2013). Se propone que una epistemología de unidad cuadrada tiene una visión más realista, como lo propone Freudenthal (Gravemeijr y Terwel, 2000).

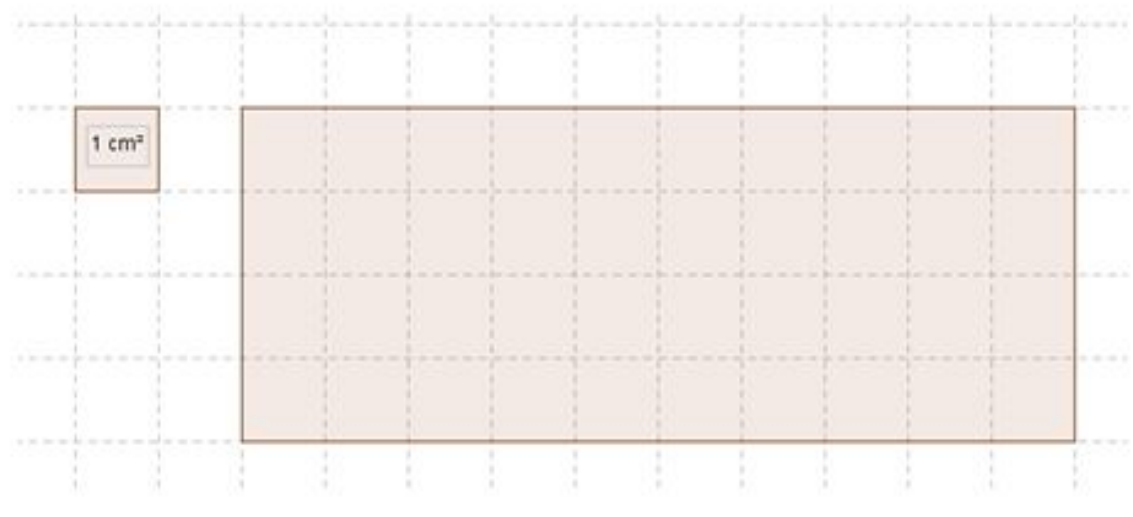

FIGURA 1

Representación de la unidad cuadrada $\mathrm{cm}^{2}$.

Fuente: Esquema de elaboración propia 


\section{Procedimiento metodológico}

Se analizaron 24 casos de estudiantes proyectando tres etapas de elaboración de una clase de matemática para enseñar el concepto de área de un cuadrilátero que fueron observados y comparados, según el modelo de la Teoría Antropológica de lo Didáctico (TAD) (Winsløw, 2009) $R E\left(a, o_{1}\right) \rightarrow R U(e, \ddot{o}) \rightarrow R E\left(e p, o_{2}\right)$. En el estudio que se propone en este artículo, interesa la etapa $R E\left(a, o_{1}\right)$ que es el estado del sistema de prácticas de un estudiante que inicia su proceso de formación en la universidad, en el curso de Didáctica de la Geometría.

\section{Tipo de investigación}

Para el logro del objetivo de la investigación, se ha adoptado un tipo de investigación cualitativo inductivo (Lamoureux, 2006; Paillé y Mucchielli, 2013; Quivy y Campenhoudt, 2006; Rodríguez, Gil, y García, 1999) más cercana a la descripción y reflexión del problema que convoca, lo cual conlleva que para este tipo de propuesta, "en lugar de una revisión de la literatura, parece más apropiado de hablar de un examen del problema que permita crear un espacio para la reflexión, la intuición, la meditación, la especulación” (Paillé y Mucchielli, 2013, p. 135). Además, esta investigación es de tipo concepción subjetiva pues hay factores que acompañan al análisis de la información y que están relacionados con la existencia de una teoría implícita para el análisis de datos; con la influencia de estereotipos sociales; con la complejidad y variabilidad de los fenómenos y con el punto de vista personal del investigador (Lamoureux, 2006; Paillé y Mucchielli, 2013; Quivy y Campenhoudt, 2006; Rodríguez et ál., 1999).

\section{Operacionalización metodológica, procedimiento de recogida de información, validez de la investigación}

Se trabajó con una muestra no probabilística, accidental y voluntaria (Lamoureux, 2006) de 24 estudiantes que ingresaron a primer año de la carrera de Pedagogía en Educación Media en Matemática, y que accedieron a participar en esta investigación. De acuerdo con los propósitos de este estudio, para observar el estado inicial de la herencia cultural didáctica del estudiantado, se les solicitó que planificaran una clase para enseñar el concepto de área de un cuadrilátero al inicio del curso de Didáctica de la Geometría (Facultad de Educación, 2011). La planificación es elaborada en el formato que ellos consideran adecuado; y que es entendido como el que corresponde a lo que ellos conocen y es parte de su sistema de prácticas inicial, de su herencia cultural didáctica. Con la planificación de la clase, se establece el estado inicial del sistema de prácticas, en lo que respecta a la enseñanza del concepto de área señalado. Esta investigación presenta específicamente los resultados de esa planificación de clase que efectúa el estudiantado. Se cautela la validez observando la coherencia interna, considerando que el objetivo de la investigación está asociado a dar cuenta de la herencia cultural didáctica que poseen los y las estudiantes y para obtener esa información, se les solicita planificar una clase para enseñar el concepto de área. Respecto de la generalización de los resultados y la validez externa, esta no se cautela pues la muestra no es representativa de la población (Lamoureux, 2006).

\section{Protocolo de análisis de los datos}

El análisis se ejecutó a través de codificación al comparar la planificación del estudiantado con elementos del Protocolo de Gestión de Aula basado la TSD de Brousseau (1986). En la siguiente Tabla 2 se presenta la estructura de códigos propuestos, en coherencia con el marco teórico: 
TABLA 2

Códigos para el análisis de la herencia cultural didáctica

\begin{tabular}{lll}
\hline Códigos 1er nivel. & Códigos 2do nivel. & Códigos 3er nivel. \\
\hline Momentos de la clase & Presenta momentos Sin momentos. & \\
\hline Contrato Pedagógico & Saludo Verificar la asistencia Instrucción Señala el objetivo & \\
& Reorganización de la posición del alumnado participante. & \\
\hline Concepto de área & Algoritmo Unidad Cuadrada Ausente & Didáctica Adidáctica Tradicional \\
\hline Relación Didáctica & Situación & Acción Formulación Validación Institucionalización \\
\cline { 2 - 3 } & Tipos & \\
\cline { 2 - 3 } & PISA (tipo de situación de acción) & \\
\hline Coprofesor - Alumno Alumno - Profesor Alumno - Alumno & \\
\hline Devolución & Concreto Pictórico Simbólico. & \\
\hline
\end{tabular}

Nota: Elaboración propia

\section{Codificación}

El proceso de análisis de las respuestas y asignación de categorías se desarrolló siguiendo el paradigma de la concepción subjetiva de Paillé y Mucchielli (2013), y usando el software QDAminer 4.1.16, y con el siguiente orden. La primera categorización se hizo considerando la respuesta asociada al contrato pedagógico y luego al concepto de área, que tiene tres posibles respuestas, algoritmo, unidad cuadrada o no la señala. La siguiente codificación que se elaboró fue la relación didáctica que conlleva tres posibilidades, didáctica, adidáctica, tradicional. Si la codificación era tradicional, entonces se decidía analizar solo la propuesta COPISI y la devolución, dejando fuera los aspectos de la TSD, pues un proceso de enseñanza tradicional no se fundamenta en el paradigma necesariamente. En cambio, si la situación observada era didáctica, se continuaba analizando el proceso fundamentado en la TSD. En la Figura 2 se presenta el diagrama de flujo para el proceso completo de análisis.

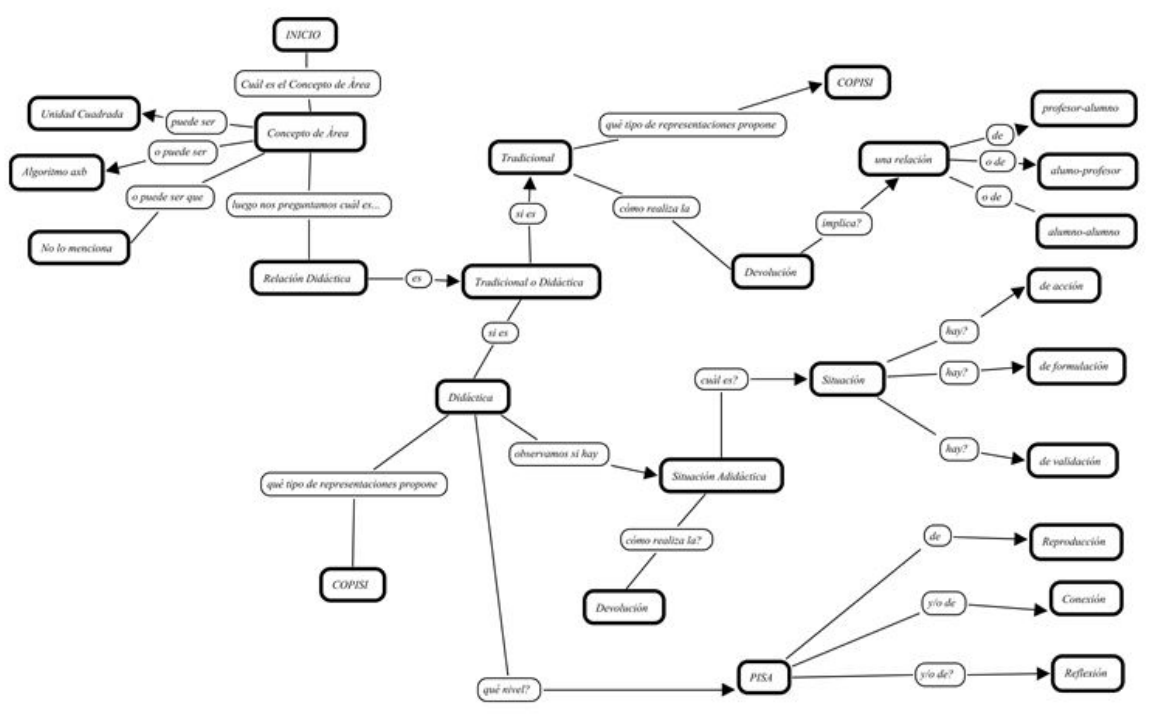

FIGURA 2

Diagrama de flujo del análisis de las clases iniciales desde la TSD

Fuente: Elaboración propia 


\section{RESULTADOS Y ANÁLISIS}

El análisis considera la observación de frecuencias porcentuales de las actividades que ocurren en la propuesta de planificación elaborada por el estudiantado, antes de iniciar el proceso de formación en didáctica de la geometría. La cantidad de estudiantes inicial que participó es de 24, que corresponde a la totalidad de la nómina de estudiantes de este curso, así este es el número que define el 100\% para el cálculo de las frecuencias.

\section{Elementos del Protocolo de Gestión de Aula}

Etapas de la Clase: Uno de los primeros elementos que se debiera considerar en la planificación de una clase, es lo que se denomina momentos de una clase, es decir, una estructura resumida que ordena las actividades. En este trabajo, se ha decidido que esa estructura debiese explicitarse con etapas como inicio, desarrollo y cierre que es la propuesta del Ministerio de Educación de Chile (MINEDUC, 2012). Los resultados muestran que los dos tercios del total de estudiantes sí consideran los momentos de la clase, relatando de esta manera una cierta preocupación o atención a dividir la clase en períodos específicos, y en que en cada uno de ellos se dan situaciones de enseñanza propias de una gestión de clase más ordenada o estructurada. El tercio restante no presenta ninguna reflexión sobre la necesidad de establecer algún tipo de etapa, no consideran el contrato pedagógico o momentos para las situaciones de acción o formulación, menos aún la presencia de procesos de devolución.

Contrato Pedagógico: Otro elemento que resulta relevante de observar es la presencia de las normas e instrucciones propuestas por el profesorado y que él considera necesarias para propiciar un ambiente de enseñanza, lo cual se denomina Contrato Pedagógico. En este caso se ha propuesto que debiesen existir cinco aspectos que de manera natural y tradicional ocurren al inicio de la clase, aspectos que son impulsados por la organización administrativa de la escuela. En la Tabla 3 se presentan los aspectos señalados con sus respectivas frecuencias porcentuales.

TABLA 3

Presencia de aspectos del Contrato Pedagógico

\begin{tabular}{llllll}
\hline & Saludo & $\begin{array}{l}\text { Verificar la } \\
\text { asistencia }\end{array}$ & Instrucción & $\begin{array}{l}\text { Señala el } \\
\text { objetivo }\end{array}$ & Reorganización \\
\hline $\begin{array}{l}\text { Contrato } \\
\text { Pedagógico }\end{array}$ & $45,8 \%$ & $8,3 \%$ & $8,3 \%$ & $20,8 \%$ & $0,0 \%$ \\
\hline
\end{tabular}

\section{Nota: Elaboración propia}

En la observación se da cuenta de la presencia del saludo protocolar; y más del $50 \%$ no lo declara, esto resulta interesante pues el saludo es parte de la formación valórica del estudiantado. Para el 20,8\% del alumnado, designar el objetivo al inicio de la clase es importante, aunque pareciera que señalarlo no tendría consecuencias en el aprendizaje pues este no elabora una actividad reflexiva sobre el objetivo. Pareciera que evidenciar el objetivo por parte del profesorado es más una actividad protocolar administrativa que de implicancias didácticas.

Tratamiento del concepto de área o base epistemológica: Para orientar el proceso de enseñanza del concepto área de un cuadrilátero, se debe conocer el concepto y definirlo desde una visión epistemológica. Se han propuesto tres formas de clasificar la forma en que el estudiantado define el concepto de área; como algoritmo, como unidad cuadrada, o no la presenta. La mayor parte del estudiantado, el 54,2\%, presenta para la enseñanza el concepto de área como el algoritmo tradicional área $=a \times b$. Llama la atención que un quinto del alumnado, el 20,8\%, lo presente como unidad cuadrada pues no es común, de acuerdo con las entrevistas 
previas efectuadas a docentes. Un resultado interesante es que el $25 \%$ de los y las estudiantes no presenta ninguna definición formal, lo que resulta relevante pues no se comprende que la planificación de una clase no considere justamente la definición conceptual de área.

Tipo de Relación Didáctica: En este proyecto se han establecido dos tipos de formas de gestión de aula, una tradicional y la otra vinculada a la TSD, que es parte de la Relación Didáctica. En la Tabla 4 se muestran los resultados para ambos tipos de gestión. Para establecer la herencia cultural didáctica inicial del estudiantado que empieza su formación, se han clasificado las respuestas justamente en la dicotomía señalada. Además, se ha subdividido la categoría Relación Didáctica en situación didáctica y adidáctica, pues se detecta en las propuestas del estudiantado que puede haber una situación didáctica pero no se presenta la situación adidáctica.

TABLA 4

Tipo de relación didáctica: situación didáctica, adidáctica, o situación tradicional

\begin{tabular}{|c|c|c|c|c|}
\hline & Situación didáctica & $\begin{array}{l}\text { Situación adidáctica (considera que sí } \\
\text { declaró la situación didáctica) }\end{array}$ & Situación tradicional & $\begin{array}{l}\text { Situación adidáctica (considera } \\
\text { que declaró una situación } \\
\text { tradicional) }\end{array}$ \\
\hline stión de aula & $25,0 \%$ & $100 \%$ & $75,0 \%$ & \\
\hline
\end{tabular}

Nota: Elaboración propia

Respecto de la presencia de una situación didáctica y una situación tradicional, el 25\% del total de estudiantes muestra la primera opción. Ellos expresan actividades vinculadas a que el alumnado desarrolle por sí mismo ciertas situaciones o ejercicios, es evidente la presencia del paradigma $<s-\mathrm{m}\rangle$. Considerando que la situación adidáctica se da posteriormente a la situación didáctica, se observa que los seis estudiantes de aquellos que proponen una situación didáctica presentan la situación adidáctica; es decir el 25\% del total de 24 estudiantes presentan una relación didáctica.

En oposición, aquellos que manifiestan una situación tradicional, el 75\%, lo hacen justamente desde una relación vertical en que el profesorado impone las actividades. Comentarios como "presentar fórmulas para determinar área y demostrar cómo llegar a ellas" (estudiante 09, comunicación personal, 11 de marzo de 2017). "Primero que todo, daré una breve definición" (estudiante 16, comunicación personal, 11 de marzo de 2017). "Comienzo explicando que el área es todo lo que cubre el cuadrilátero" (estudiante 17, comunicación personal, 11 de marzo de 2017). Hay un caso que representa muy bien la relación didáctica tradicional, "se saluda...luego de esto se da una pequeña definición de qué es un cuadrilátero" (profesor 13, comunicación personal, 11 de marzo de 2017). Luego el mismo estudiante 13 subraya: "pondremos la fórmula para sacar el área con un ejemplo resuelto por mí, explicando cada paso qué se hace, así los alumnos pueden entender con claridad" (estudiante 13, comunicación personal, 11 de marzo de 2017). El caso de este estudiante es interesante pues tiene una herencia cultural didáctica muy tradicional, en que el profesorado al explicar logra el aprendizaje del alumnado; práctica muy opuesta al paradigma $<s-m>$. Este tipo de relación es bien habitual y con una presencia explícita.

El estudiantado tradicional manifiesta en su rol de profesor tener el control de todo lo que está ocurriendo e imponer las respuestas al alumnado; se observa explícitamente la presencia del efecto Topaze (Brousseau, 2013; Johsua y Dupin, 2005).

Relación didáctica versus Epistemología del concepto de área: Cuando se analiza la información cruzada, hay dos estudiantes que presentan una relación didáctica tradicional, pero poseen una epistemología del concepto de área de unidad cuadrada, esto los hace un caso interesante, pues el concepto de unidad cuadrada promueve una relación didáctica y que la relación $\langle s-m\rangle$ se haga presente. Se propone que para este caso de estudiantes modificar su proceso de relación didáctica en un proceso de formación de docentes sería bastante simple. 
En el caso opuesto, hay 11 estudiantes que presentan una relación didáctica tradicional y una epistemología del concepto de área algorítmica, lo que equivale al 45,8\% del total de estudiantes. Esta herencia cultural didáctica del estudiantado es la esperada en aquellos que ingresan a la UCSC a formarse como docentes de matemáticas, pues es lo que se ha observado como una práctica de enseñanza regular en la entrevista a docentes de matemáticas del sistema educativo chileno.

Presencia de elementos de la Gestión de Aula: Se ha propuesto un modelo de gestión de aula que incluye las situaciones de acción, formulación, validación e institucionalización. En la Tabla 5 se presentan los resultados asociados a su presencia o ausencia. Dado que estos elementos son parte de una Relación Didáctica, se ha optado por analizar la presencia de estos elementos solo en aquellos y las estudiantes que manifiestan una relación didáctica no tradicional.

TABLA 5

Presencia de las situaciones de acción, formulación, validación e institucionalización

\begin{tabular}{ccccc}
\hline & Acción & Formulación & Validación & Institucional. \\
\hline Tipo de situación & $12,5 \%$ & $8,3 \%$ & $8,3 \%$ & $16,7 \%$ \\
\hline
\end{tabular}

Nota: Elaboración propia

En el análisis de los resultados se observa que tres estudiantes presentan situación de acción, habiendo previamente expresado una situación didáctica. Las preguntas que se ejecutan son: “¿Cuál es el área de una sala de clases?" (estudiante 18, comunicación personal, 11 de marzo de 2017). "Identificar las partes de una figura" (estudiante 12, comunicación personal, 11 de marzo de 2017). "Si el perímetro de un terreno cuadrado es la suma de su contorno" (estudiante 22, comunicación personal, 11 de marzo de 2017). Más aún, cada uno de los tres estudiantes, luego de la situación de acción, presenta un relato que implica la relación. En el caso del estudiantado 22, declara: "posteriormente al planteamiento del problema, se le estimula para que razone cómo calcular el área” (estudiante 22 comunicación personal, 11 de marzo de 2017), por lo que hay una fuerte cercanía hacia la devolución. En el caso del estudiantado 12, recalca: "posteriormente pregunta a sus estudiantes qué partes pueden ser identificadas en dicha figura. Si bien las respuestas pueden ser variadas, deben lograr identificar el largo, el ancho" (estudiante 12, comunicación personal, 11 de marzo de 2017) y posteriormente declara "luego de hacer que los y las estudiantes participen en su propia confección de conocimiento, el docente da la respuesta final" (estudiante 12, comunicación personal, 11 de marzo de 2017). Estos tres casos aparecen como interesantes de ser observados en una posterior investigación del recorrido del proceso de formación, ya que se puede suponer que dado que la herencia cultural didáctica es cercana a la TSD, sus prácticas pedagógicas tendrán poca o ninguna influencia de una Situación Tradicional, facilitando así la formación de un profesor de matemática en coherencia con el paradigma .

Respecto de las situaciones de formulación, se da explícitamente a través de la declaración "el profesorado organiza a los alumnos en grupos para que resuelvan el siguiente problema" (estudiante 18, comunicación personal, 11 de marzo de 2017). Este estudiante es el único estudiante que muestra una presencia de la situación de acción y de formulación. El resto de los observados, solo situación de acción o de formulación. Hay un caso que señala para la situación de formulación: "desarrollar entre todos los alumnos el cuestionario y hacer un trabajo con maquetas y esqueletos, demostrando cómo pueden encontrar el área de un cuadrilátero" (estudiante 23, comunicación personal, 11 de marzo de 2017). Es un caso interesante pues declara una situación de formulación de acuerdo con el paradigma .

En el caso de situación de validación, dos estudiantes del total de 24 la expresan, "para finalizar, una pequeña actividad grupal en la cual cada grupo tenga por lo menos a un alumno que comprenda a cabalidad el conocimiento entregado, y así producir eficiencia en la conformación de dichos grupos, revisamos la actividad en forma general" (estudiante 24, comunicación personal, 11 de marzo de 2017). "Pediría algún voluntario que quisiera explicar a qué resultado llegó” (estudiante 04, comunicación personal, 11 de marzo de 2017). 
Ambos casos no parecen ser muy conscientes de la importancia de la situación de validación, pensando en el hecho que el trabajo en grupo y la posterior presentación de resultados frente al curso es una actividad, según nuestro punto de vista, bastante utilizada en las prácticas de enseñanza en la escuela en general.

En el caso de la institucionalización, cuatro estudiantes de aquellos que proponen una situación didáctica, plantean una institucionalización. Un caso interesante es el que presenta una situación didáctica que es "calcular el área de la cancha para saber cuánto césped voy a necesitar para completar en su totalidad la cancha" (estudiante 17, comunicación personal, 11 de marzo de 2017). Luego declara: "les hago notar que el resultado (u. de m. $)^{2}$ con que a su vez les hago cuadricular la cancha en cuadrados, para que absorban el hecho de que son unidades de medida al cuadrado" (estudiante 17, comunicación personal, 11 de marzo de 2017). Este estudiante resulta un caso interesante, pues su propuesta de planificación es muy cercana a la propuesta de protocolo de gestión de aula que se plantea en esta investigación.

Continuando con la institucionalización, entre los casos que han planteado una situación tradicional, es claro que en ellos sí se manifiesta la institucionalización. Tal es el caso que declara: "haría una breve definición (simple) y daría ejemplos para familiarizarlas con las figuras como cuadrados, rectángulos, rombos, romboide u otros" (estudiante 19, comunicación personal, 11 de marzo de 2017). O el caso “enseñaré y aprenderán el área de un cuadrilátero, tomaremos los casos de diferentes tipos de cuadriláteros (cuadrados, rectángulos, y cuadriláteros irregulares, ángulos diferentes a $90^{\circ}$ ). Empiezo por recordar qué es un cuadrilátero, muestro 5 figuras en la pizarra" (estudiante 24, comunicación personal, 11 de marzo de 2017). O el caso "suponiendo que ya han pasado el concepto de área, y para recordar más profundamente hacemos unas pequeñas definiciones con conceptos previos, allí colocamos: área, cuadrado, rectángulo, rombo, romboide" (estudiante 14, comunicación personal, 11 de marzo de 2017). En todos los casos de situación didáctica tradicional, se repiten o aparecen frases relacionadas a institucionalizar el contenido al inicio de la clase.

Respecto de los tipos de situación de acción definidos a través de las competencias PISA, se presentan 6 estudiantes que de forma explícita dan presencia a través de preguntas a alguno de los niveles, por ejemplo un caso a nivel de reproducción pregunta: “¿Qué es un cuadrilátero? ¿Cuáles son sus características?, dentro de la sala de clases ¿podemos encontrar algo que se asemeje a uno?” (estudiante 11, comunicación personal, 11 de marzo de 2017). Hay un caso que presenta evidencia del nivel reflexión, cuando advierte "pido para la próxima clase los alumnos lleguen con un ejemplo cotidiano de la necesidad de calcular el área de dicha figura geométrica" (estudiante 17, comunicación personal, 11 de marzo de 2017). Cuatro casos del total presentan evidencias de nivel conexión, "¿cuál es el área de una sala de clases, si sus medidas son $4 \mathrm{~m}$ de ancho y $5 \mathrm{~m}$ de largo?” (estudiante 18, comunicación personal, 11 de marzo de 2017). El resto de los y las estudiantes no presenta evidencia. Al ser principalmente tradicionales, solo muestran lo que ellos y ellas harían o señalarían al alumnado.

Respecto de COPISI, y considerando el protocolo establecido que se explica en el proceso de análisis de la Figura 2, se obtiene que el 89\% de los casos presentan un tipo de representación simbólica, y ningún caso presenta una representación concreta o pictórica. Con esto se evidencia lo supuesto que la mayoría de los y las estudiantes tiene una herencia cultural didáctica asociada a los algoritmos.

En el caso de la devolución, la Tabla 6 muestra los valores asociados su presencia.

TABLA 6

Presencia de la devolución

\begin{tabular}{ccc}
\hline Tipo de situación & Profesor Alumno & Alumno Profesor \\
\hline Didáctica & $4 \%$ & $21 \%$ \\
\hline Tradicional & $63 \%$ & $13 \%$ \\
\hline
\end{tabular}


Como se observa, la mayor presencia de la devolución se da en la situación didáctica en que el alumnado dialoga con el profesorado. La relación unidireccional de profesor alumno con posibilidad de presencia del efecto Topaze se da en la situación tradicional.

\section{Análisis dendograma}

La construcción del dendograma permite analizar relaciones entre los conceptos. Un primer análisis se hace respecto de la Situación Tradicional. Es en el estudiantado que ha mostrado una planificación opuesta al paradigma, sobre los cuales el proceso de formación de docentes de matemáticas debiera producir cambios importantes y relevantes, basados en la TSD. En la Figura 3 se muestran cinco conglomerados asociados a la situación didáctica tradicional:

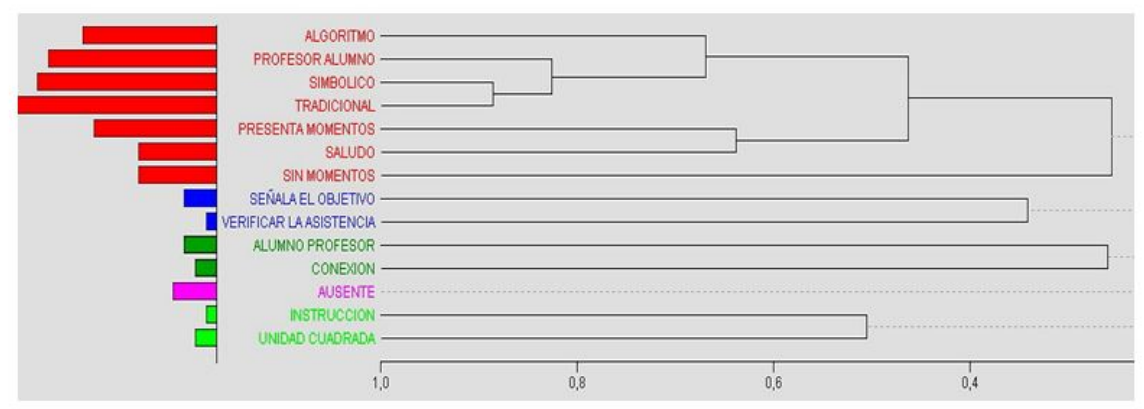

FIGURA 3

Dendograma de la situación didáctica tradicional con 5 conglomerados

Fuente: Elaboración propia con QDAminer 4.1.16.

$\mathrm{Al}$ observar, llama la atención que la categoría más alejada corresponde a unidad cuadrada, por sobre las otras variables. Se observa que la mayor relación se da entre el tipo de epistemología del concepto de área y el tipo de situación didáctica; es decir el estudiantado que manifiesta un tipo de enseñanza tradicional, tienen una fuerte concepción de área vinculado al algoritmo, a lo simbólico, a la aplicación directa de la fórmula en una situación; lo tradicional y lo simbólico están juntos. Además, esto se destaca al observar que la epistemología del concepto de área como unidad cuadrada aparece muy distante de una relación didáctica tradicional.

Otro aspecto del dendograma que resulta relevante es el hecho de hacerse presente la devolución en la relación alumno profesor, es decir el profesorado propicia una relación dialógica con participación del alumnado, cuando se presentan situaciones de acción de tipo conexión, lo que permitiría que el alumnado desarrolle capacidades de resolver ejercicios o tareas de matemáticas vinculadas al concepto de área, abordando "situaciones no rutinarias pero en marcos familiares o casi familiares" (OCDE, 2006, p. 105). Así, la devolución (Brousseau, 2013) se produce con mayor presencia cuando el profesorado promueve una conexión entre la matemática y la realidad; o cuando promueve un vínculo entre los ejercicios de matemáticas simbólicos y el mundo real.

En el análisis global del dendograma, se comprueba que hay una fuerte presencia del paradigma de la situación didáctica tradicional, en que: la conjunción de una epistemología algorítmica de los conceptos matemáticos; una relación vertical profesor - alumno; una fuerte presencia de tareas o actividades de tipo simbólico, hacen que el alumnado no desarrolle las capacidades fundamentales en los actuales procesos de aprendizaje matemáticos declarados en las políticas fundantes PISA; "evaluar en qué medida los alumnos pueden ser considerados unos ciudadanos reflexivos e informados y unos consumidores inteligentes" (OCDE, 2006, p. 74), y en los propios programas de estudio chilenos que determinan que el propósito formativo de la matemáticas es: "enriquecer la comprensión de la realidad, facilitar la selección de 
estrategias para resolver problemas y contribuir al desarrollo del pensamiento crítico y autónomo en todos los alumnos" (MINEDUC, 2012, p. 145) y continúa señalando que: "aprender matemática proporciona herramientas conceptuales para analizar la información cuantitativa presente en las noticias, opiniones, publicidad y diversos textos, aportando al desarrollo de las capacidades de comunicación, razonamiento y abstracción" (p. 145). Los resultados permiten proponer que una práctica de enseñanza tradicional no propicia lo indicado en relación con impulsar el desarrollo del pensamiento intuitivo y la reflexión sistemática.

\section{ConClusión}

La investigación muestra que aquellos y las estudiantes que tienen una concepción epistemológica de área como algoritmo a $\mathrm{x}$ b, presentan una planificación tipo situación didáctica tradicional, y en oposición, aquellos y las estudiantes que presenta una epistemología del concepto de área como unidad cuadrada, presentan una planificación de clase con una situación didáctica fuertemente vinculada al paradigma .

Se concluye que el estudiantado encuestado presenta una herencia cultural didáctica vinculada a como sus docentes le enseñaron en la escuela (Chevallard, 1999; Winsløw, 2009, 2013). De acuerdo con los resultados, la mayoría de estudiantes consultados presentan una epistemología del concepto de área como algoritmo tradicional a $\mathrm{x}$ b, y el profesorado la impone al alumnado unidireccionalmente y no propicia una actividad dialógica. Además, direcciona la actividad del profesorado a plantear situaciones de tipo reproductivo (OCDE, 2006) con representación simbólica. La ausencia de actividades de reflexión y de situaciones de tipo concreta o pictórica es notoria y relevante.

Una minoría de estudiantes tiene una epistemología del concepto de área de unidad cuadrada. Dado lo propuesto por Winsløw (2009), se propone que el personal docente de matemática en la escuela de estos estudiantes, presenten esta forma de determinar el área, lo cual se muestra como positivo pues dicha presentación, de acuerdo con los resultados de este trabajo, conlleva procesos dialógicos más elaborados que aquella que muestra el algoritmo tradicional, que solo se impone y es característico de una enseñanza tradicional. Existe presencia de la relación en la herencia cultural didáctica de estos estudiantes.

Un 25\% de la muestra de estudiantes no entrega información suficiente para definir la forma en que definen el concepto de área; se desconoce la razón. Esto permite suponer, desde el punto de vista didáctico, que este estudiantado no ha asumido la importancia de la presencia de la definición conceptual de área, o al menos, no es parte aún de su herencia cultural didáctica. Al sumar la visión algorítmica y la ausencia del concepto da un total de $75 \%$, lo cual implica que son estudiantes que no tienen una formación pertinente en el concepto de área.

Respecto del modelo de enseñanza del concepto de área, 18 de los y las estudiantes consultados presentan una herencia cultural didáctica del tipo idealista platónica. Esto, de acuerdo con los resultados implica una mayor presencia de ejercicios de reproducción y simbólicos. Esta forma de enseñanza va en oposición con la propuesta de formación basada en la TSD. Desde el punto de vista de la enseñanza del concepto de área, proponer la fórmula a $\mathrm{x}$ b por sobre la unidad cuadrada acota la comprensión del concepto de área, pues para el alumnado es complejo relacionar el algorítmico con el mundo real, ya que no es parte del medio del alumnado de acuerdo con el paradigma . Además, el proceso de institucionalización se presenta como una característica de la herencia cultural didáctica en el sentido de oficializar el conocimiento que el alumnado debe aprender de acuerdo con el currículo oficial establecido al inicio de la clase. Esto implica un 63\% de casos en que la relación es unidireccional de profesor alumno con posibilidad de presencia del efecto Topaze y ausencia de devolución que posibilite la presencia del paradigma. La misma enseñanza tradicional implica una ausencia de las situaciones de formulación o validación, que minimiza la presencia de la devolución. Esto permite mencionar que se deja de promover la relación entre el concepto de área y el mundo real o realista (Gravemeijr y Terwel, 2000), y en el paradigma, el aprendizaje se produce por adaptación al medio 
en presencia de la situación adidáctica; y uno de los elementos de la TSD que la propicia es la devolución. En esta investigación se da cuenta que existe mayor presencia de la devolución cuando el estudiantado propone un tipo de ejercicio matemático de área aplicado al mundo real, en cierta manera como lo plantea "el ciclo de la matematización" (OCDE, 2006, p. 99).

Se propone considerar en los procesos de formación de docentes tener una fuerte presencia del mundo real asociado a conceptos matemáticos, así como destacar la presencia de situaciones de tipo reflexivo (PISA) y las diversas formas de representación COPISI. Además, asumir una cierta labor de perfeccionamiento docente a docentes que ya se encuentren en ejercicio profesional para que ellos mismos recuerden o actualicen sus procesos de enseñanza de sus clases de matemáticas en la escuela.

\section{Agradecimientos}

Esta investigación fue financiada a través del proyecto DIN 04/2013

\section{REFERENCIAS}

Acosta, M., Monroy, L. y Rueda, K. (2010). Situaciones a-didácticas para la enseñanza de la simetría axial utilizando Cabri como medio. Revista Integración. Universidad Industrial de Santander, 28(2), 173 - 189.

Ávalos, B. (2004). La Formación Docente Inicial en Chile. Santiago: Unesco.

Bacon, L. (2009). Construction négociée par la triade de formation en stage d'un savoir-enseigner les mathématiques au primaire. (Tesis Doctoral). Universidad de Montreal, Canadá. Recuperada de https://papyrus.bib.umontreal.c a/xmlui/handle/1866/5328.

Ball, D., Hill, H. C. y Bass, H. (2005). Knowing Mathematics for Teaching: Who Knows Mathematics Well Enough To Teach Third Grade, and How Can We Decide? American Educator, 29(1), 14-17.

Ball, D., Thames, M. y Phelps, G. (2008). Content Knowledge for Teaching: What Makes It Special? Journal of Teacher Education, 59(5), 389-407.

Brousseau, G. (1986). Fondements et méthodes de la didactiques des mathématiques. Recherches en Didactique des Mathématiques, 7(2), 33-115.

Brousseau, G. (2007). Iniciación al estudio de la teoria de las situaciones didácticas. Argentina: Libros del Zorzal.

Brousseau, G. (2013). Glossaire de quelques concepts de la théorie des situations didactiques en mathématiques. Recuperado de https://bit.ly/2JJ3B48

Clemens, S. R., O'daffer, P. G. y Cooney, T. J. (1998). Geometría. México: Addison Wesley.

Chavarría, J. (2006). Teoría de las Situaciones Didácticas. Cuadernos de investigación y formación en educación matemática, 1(2), 1-10.

CNA-Chile. (19 de diciembre de 2018). Comisión Nacional de Acreditación. Recuperado de https://www.cnachile.cl

Chevallard, Y. (1999). El análisis de las prácticas docentes en la teoría antropológica de lo didáctico. Recherches en Didáctique des Mathématiques, 19(2), 221-266.

D'Amore, B. (2005). Bases filosóficas, pedagógicas, epistemológicas y conceptuales de la Didáctica de la Matemática. México: Reverté.

D'Amore, B. (2011). Didáctica de la Matemática. Bogotá: Cooperativa Editorial Magisterio.

D'Amore, B., y Fandiño, M. (2002). Un acercamiento analítico al triángulo de la didáctica. Educación Matemática, $14(1), 48-61$.

Facultad de Educación. (2011). Planes y Programas Pedagogía Media en Matemática. Concepción, Chile: Ediciones UCSC.

Facultad de Educación. (2012). Programa de Estudio Geometría en el Plano y su Didáctica. Concepción, Chile: Ediciones UCSC. 
Godino, J., Batanero, C. y Font, V. (2003). Fundamentos de la enseñanza y el aprendizaje de las matemáticas para maestros. Granada, España: Universidad de Granada.

Gravemeijr, K. y Terwel, J. (2000). Hans Freudenthal, un matemático en didáctica y teoría curricular. Curriculum Studies, 32(6), 777 - 796.

Johsua, S. y Dupin, J. (2005). Introducción a la Didáctica de las Ciencias y las Matemáticas. Argentina: Ediciones Colihue.

Lamoureux, A. (2006). Recherche et méthodologie en sciences humaines. Quebec. Beauchemin.

Martínez, S. (2014). Recursospara la formación inicial de docentes de educación básica. Santiago, Chile: Proyecto Fondef D09 I1023.

MINEDUC. (2004). Reglamento sobre evaluación docente. Recuperado de https://bit.ly/2YyWhMp

MINEDUC. (2012). Programas de Estudio. Recuperado de https://bit.ly/2HwW9GA

MINEDUC. (2013). Perímetros y áreas de figuras geométricas. Santiago, Chile: Ministerio de Educación. Recuperado de https://bit.ly/2WToIVe

MINEDUC. (2018). Informativo: Valores 2018 para BRP y asignaciones de Carrera Docente. Recuperado de https:/ /bit.ly/2w3kw9n

Morales, H. (2017). La teoría de las situaciones didácticas como sustento teórico en la formación de docentes. En UCSC (Ed.). La didáctica como fundamento para la formación inicial y continua de docentes. Tendencias, enfoques $y$ avances (p. 67-88). Concepción, Chile: Editorial UCSC.

OCDE. (2006). PISA 2006. Marco de la evaluación. Conocimientos y habilidades en Ciencias, Matemáticas y Lectura. España: Santillana.

Paillé, P. y Mucchielli, A. (2013). L'analyse qualitative en sciences humaines et sociales. París: Armand Colin.

Perrenoud, P. (2000). Mobiliser ses acquis: où et quand cela s'apprend-il en formation initiale? De qui est-ce l'affaire? Recherche y formation, (35), 9-23. Recuperada de https://www.persee.fr/doc/refor_0988-1824_2000_num_35 _1_1667

Quivy, R. y Campenhoudt, L. V. (2006). Manuel de recherche en sciences sociales. París: Dunod.

Reyes, C., Dissett, L. y Gormaz, R. (2013). Geometría. Chile: Maval.

Rodriguez, G., Gil, J. y García, E. (1999). Metodología de la investigación cualitativa. Málaga, España: Aljibe.

Winsløw, C. (2009). First Years of Teaching. En R. Even y D. L. Ball (Eds.). The Professional Education and Development of Teachers of Mathematics (pp 93-101). New York: Springer.

Winsløw, C. (2013). The transition from university to high school and the case of exponential functions. EN F. Arzarello (Presidente), Eighth Congress of European Research in Mathematics Education. Conferencia llevada a cabo en el congreso CERME 8, Manavgat-Side, Antalya - Turquía.

\section{FinANCIAMIENTO}

Fuente: proyecto DIN 04/2013

Beneficiario: La herencia cultural didáctica: Un estudio relativo al concepto de área en la formación docente

\section{BY-NC-ND}

